\title{
11th Mexican Congress on Catalysis: Catalysis and Nanotechnology
}

\author{
A. Olivas $\cdot$ G. Alonso-Núñez $\cdot$ T. A. Zepeda \\ V. Petranovskii
}

Published online: 21 January 2011

(C) Springer Science+Business Media, LLC 2011

\section{Preface}

The "Mexican Catalysis Academy", founded in 1988, has organized 11 biannual conferences, mainly devoted to homogeneous and heterogeneous catalysis. The quality and quantity of the contributions presented in these conferences has increased favorably over the years, as well as the diversity of the research fields. This special issue contains a representative part of the papers presented at the 11th Mexican Congress on Catalysis, in this occasion with the theme "Catalysis and Nanotechnology", which took place in Ensenada B.C., Mexico, from June 2nd to 5th, 2009, with more than 100 contributions. Contributors, in this issue, were invited to submit their original manuscripts which were peer-reviewed according to international procedures. We are most pleased to publish in this issue the Mexican contributions for the scientific community.

The main topics in this issue have been related with the synthesis, physico-chemical characterization and catalytic performance of unsupported and supported nano-catalysts, with several nanotechnology catalytic-applications focused on various aspects of the catalysis as follows:

A. Olivas · G. Alonso-Núñez $(\bowtie) \cdot$ T. A. Zepeda ·

V. Petranovskii

Centro de Nanociencias y Nanotecnología, Universidad Nacional Autónoma de México, 22800 Ensenada, B.C, México

e-mail: galonso@cnyn.unam.mx
A. Olivas
e-mail: aolivas@cnyn.unam.mx
T. A. Zepeda
e-mail: trino@cnyn.unam.mx
V. Petranovskii
e-mail: vitalii@cnyn.unam.mx

- Production of alternative fuels, such as deoxygenation and esterification of fatty acids and hydrogen production.

- Hydroprocessing of fraction oils, such as hydrodesulfurization, hydrocracking and oxidative desulfurization of diesel.

- Photocatalytic applications for environment protection, such as degradation of alizarin red, phenol, 4-nitrophenol, 4-chlorophenol, etc.

- Surface studies of carbon adsorbed species on vanadium versus nickel and platinum single crystals.

- Hydrogenation and oxidation reactions.

The Guest Editors thank all the authors for their excellent contributions and cooperation in the submission and revision process during this edition. Our most sincere acknowledge and gratitude to all the reviewers for their assistance in refereeing the papers. Professor Sergio Fuentes Moyado, Director of the Nanoscience and Nanotechnology Center of UNAM, in Ensenada, and the Mexican Catalysis Academy A.C., are gratefully appreciated for their support in the organization of this event. Finally, we would also like to thank Professor Gabor A. Somorjai, Editor-in-chief of "Topics in Catalysis", and Springer Editorial for publishing this volume. 